\title{
Proteomics-based metabolic modeling and characterization of the cellulolytic bacterium Thermobifida fusca
}

\author{
Niti Vanee ${ }^{1}$, J Paul Brooks ${ }^{1}$, Victor Spicer ${ }^{2}$, Dmitriy Shamshurin², Oleg Krokhin², John A Wilkins², Yu Deng ${ }^{3}$ \\ and Stephen S Fong ${ }^{1 *}$
}

\begin{abstract}
Background: Thermobifida fusca is a cellulolytic bacterium with potential to be used as a platform organism for sustainable industrial production of biofuels, pharmaceutical ingredients and other bioprocesses due to its capability of potential to convert plant biomass to value-added chemicals. To best develop T. fusca as a bioprocess organism, it is important to understand its native cellular processes. In the current study, we characterize the metabolic network of $T$. fusca through reconstruction of a genome-scale metabolic model and proteomics data. The overall goal of this study was to use multiple metabolic models generated by different methods and comparison to experimental data to gain a high-confidence understanding of the T. fusca metabolic network.

Results: We report the generation of three versions of a metabolic model of Thermobifida fusca sp. XY developed using three different approaches (automated, semi-automated, and proteomics-derived). The model closest to in vivo growth was the proteomics-derived model that consists of 975 reactions involving 1382 metabolites and account for 316 EC numbers (296 genes). The model was optimized for biomass production with the optimal flux of 0.48 doublings per hour when grown on cellobiose with a substrate uptake rate of $0.25 \mathrm{mmole} / \mathrm{h}$. In vivo activity of the DXP pathway for terpenoid biosynthesis was also confirmed using real-time PCR.

Conclusions: iTfu296 provides a platform to understand and explore the metabolic capabilities of the actinomycete T. fusca for the potential use in bioprocess industries for the production of biofuel and pharmaceutical ingredients. By comparing different model reconstruction methods, the use of high-throughput proteomics data as a starting point proved to be the most accurate to in vivo growth.
\end{abstract}

Keyword: Metabolic Modeling, Flux Balance Analysis, Constraint Based Modeling, Actinomycete, Thermobifida fusca, Proteomics Profiling, Terpenoids Biosynthesis Pathway, DXP Pathway, Mevalonate Pathway, Biofuel

\section{Background}

With ongoing research in genomics, metagenomics, and bioprospecting, the breadth of novel and interesting biochemistry continues to grow. One of the current challenges associated with the large amount of data and resources available is to conduct detailed analyses to curate information to translate raw data into knowledge that provides functional insight. One computational method that facilitates metabolic analysis and dovetails well with genomic and biochemical information is genome-scale

\footnotetext{
* Correspondence: ssfong@vcu.edu

'Virginia Commonwealth University, Richmond, USA

Full list of author information is available at the end of the article
}

constraint-based modeling. While constraint-based models have benefits of being easily scalable and providing geneprotein-reaction level specificity, there are a number of limitations. One of the most fundamental problems revolves around the fact that metabolic networks are underdetermined and thus, there exist alternative flux states with different pathway usage that produce indistinguishable cellular phenotypes. This is an underlying problem with constraint-based models that impacts multiple facets of these models including the initial reconstruction (association of specific reactions with annotated 
genes) to producing simulation predictions (presence of alternate optimal solutions). In this study, we consider the metabolically under-characterized actinobacterium, Thermobifida fusca, and utilize three different methods to gain a better understanding of its metabolic network and identify preferred methodologies for network characterization.

\section{Thermobifida fusca}

Within the actinomycetes, Thermobifida fusca (aerobic, thermophilic, gram-positive) is known for its high temperature and $\mathrm{pH}$ stability as well as highly expressed cellulolytic system. The cellulolytic system is comprised of three endocellulases (Cel9B, Cel6A and Cel5A), two exocellulases (Cel6B and Cel48A) and a processive cellulase (Cel9A) [1]. Numerous studies in the past have reported on various facets of the ability of T. fusca to degrade lignocellulosic biomass. Due to the high efficiency with which T. fusca can process lignocellulosic materials, efforts have been made to clone several individual cellulase genes into Streptomyces lividians, Streptomyces albus, Bacillus subtilis [2] and Escherichia coli [3,4]. The cloned enzymes were isolated in good concentrations but failed to show similar level of cellulolytic activity as is found in $T$. fusca. This may be due to the complexity of cellulose degradation systems that are not defined by a few genes but is an intertwined network of various enzymes $[1,5]$. Hence, it is suggested that the best way to fully utilize the cellulolytic capabilities of T. fusca may be to develop $T$. fusca rather than trying to move its cellulases into other systems by heterologous expression.
Actinomycetes have historically been involved in the biological production of a variety of antifungals, antibiotics, and chemotherapeutics. Some of these compounds include siderophores [6], polyketides [7,8] and terpenes $[9,10]$. As a cellulolytic actinomycete, $T$. fusca may present an interesting opportunity for consolidated bioprocessing CBP (Figure 1) of raw lignocellulosic material to valueadded biobased products.

The ability to produce chemicals of industrial importance using inexpensive lignocellulosic biomass has been a recent focus for microbial systems. For T. fusca, the sequencing of its genome by Department of Energy (DOE) in 2005 sets up a milestone towards understanding this industrially applicable microbe [11]. Besides proving an excellent host microbe for biofuel production [12], it also showed success towards utilization of untreated (without any preprocessing) lignocellulosic material. This is a promising development toward making use of the cellulolytic capabilities of this microbe to reduce the complex multi-step bioprocess to a CBP [13].

Different approaches have been taken in the past in trying to develop a consolidated bioprocess. One approach is to utilize the cellulolytic capabilities to well-established model organisms, such as E. coli and S. cerevisiae [1]. This approach seeks to allow for direct use of lignocellulosic biomass as a starting point, but leverages the knowledge and tools available for well-characterized organisms. The alternative approach is to characterize and develop poorly-characterized cellulolytic organisms. Thus, high levels of cellulose processivity can be achieved, but the

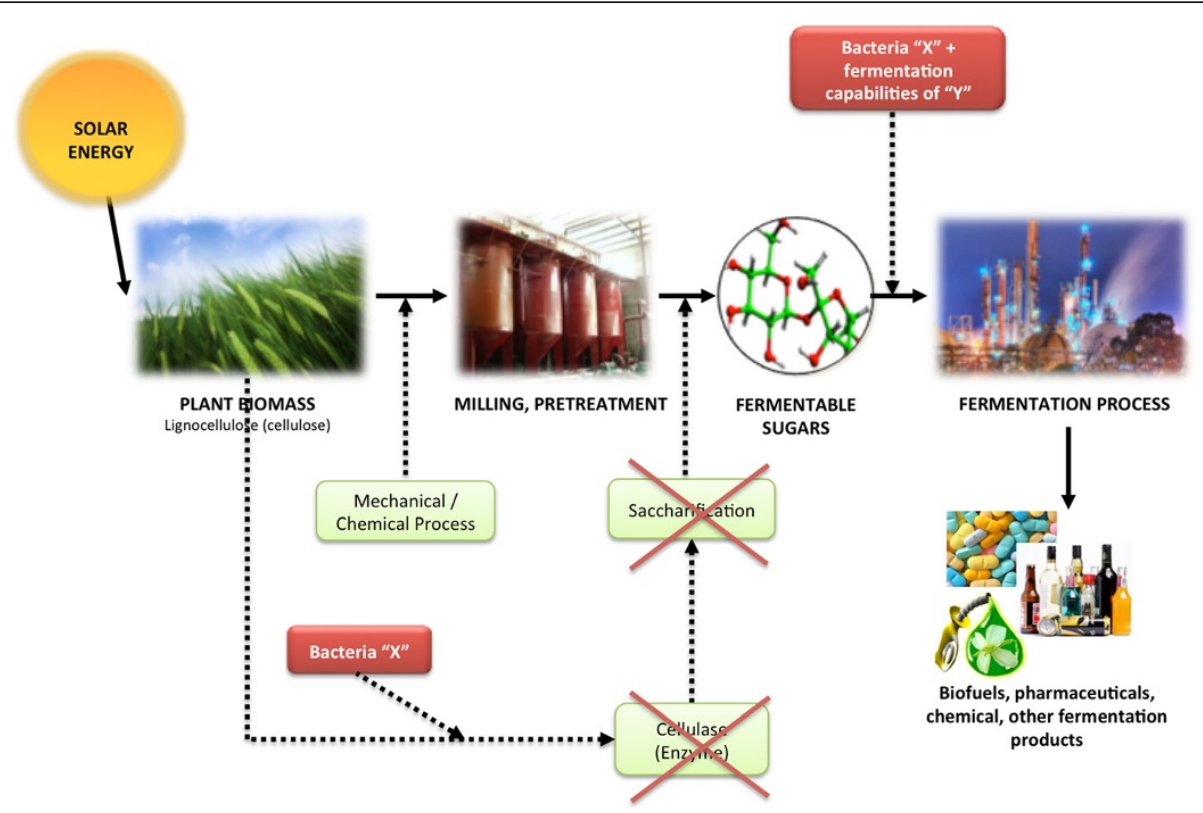

Figure 1 The prospective use of cellulolytic microbes such as T. fusca may help in reducing the multiple steps towards the fermentation bioprocess there by reducing the need of pretreatment of plant biomass to feed into an industrial process as a source of carbon. Bacteria- $X$ denotes the cellulolytic capabilities of microbes such as T. fusca and Bacteria-Y denotes the fermentations potentials of industrial microbes 
challenge is to develop the knowledge and tools to a sufficient level that metabolism can be designed and altered in a directed fashion.

In studying $T$. fusca, we believe that it can be developed into a facile cellulolytic system for consolidated bioprocessing. In 2011, Deng and Fong established a genetic modification protocol for genetic engineering of T. fusca and demonstrated this system through the production and optimization of propanol [12]. The initial developments in the characterization of T. fusca are listed in Table 1. Following along the same lines, we continue to further understand the other capabilities of robust cellulolytic system of actinomycete for use in manufacturing industries. Thus, our group aims to present a systems level understanding of metabolic network of T. fusca.

\section{Metabolic models}

With the availability of genomic sequences, it has become possible to use genome annotation and biochemical information to reconstruct cellular metabolic networks [14]. These models can be used for simulating the living state of bacteria, if operated under the defined constraints and boundary conditions. There are various algorithms such as FBA - flux balance analysis [15,16], MOMA - minimization of metabolic adjustments [17], ROOM - regulatory on-off minimization [18] and MCA - metabolic control analysis that are currently used for the purpose of running computational simulations of these models [19]. In the current study, we used FBA, which is based on linear programming, to simulate and optimize T. fusca model developed in this study for biomass production (growth).

\section{T. fusca model and FBA}

Whole genome sequence and annotation of T. fusca [11] was used to build a genome-scale metabolic reconstruction to understand the underlying metabolic pathways and network in T. fusca $[11,12,20]$. The reaction database used for drafting the model includes but is not limited to sources such as KEGG [21,22], BiGG [23], rBioNet
[24], UniProt [25] and MBRole [26]. Information from disparate databases were tabulated to form an in-house reaction database as illustrated in Figure 2.

The process of compiling biochemical reactions leads to the formulation of a draft model of T. fusca, however, at this stage the represented metabolic network inevitably is incomplete and has numerous metabolic gaps. Thus, any initial model that is generated undergoes a gap-filling step. For our models, gap-filling was initially performed using a computational gap-filling algorithm [27-30]. After gap-filling, simulations can be run by specifying input constraints such as substrate uptake rate or oxygen uptake rate. Once the model provides a framework to understand the cellular process, it can be used to find target for focused metabolic engineering to yield products of biotechnological value [31]. The most widely-used algorithms for design and simulation of genome-scale constraint-based metabolic models such as OptKnock [32], OptForce [33], EMiLio [34] are based on flux distribution and flow through chemically balanced reactions (FBA). Flux balance analysis (FBA) uses linear programming to optimize the objective function as follows:

\section{Maximize : Z \\ Subject to : $\mathrm{Sv}=0$, $a_{\mathrm{i}} \leq v_{\mathrm{i}} \leq b_{\mathrm{i}}$ for all reactions $i$,}

where, $Z$ is the flux through objective function (biomass production and product optimization), S: stoichiometry of the reactions represented as matrix, $v$ is reaction flux vector, $a_{i}$ and $b_{i}$ are the constraints placed on the flux $v_{i}$ of the reaction $i$ [35].

Even after compiling biochemical information and gap-filling a model, there often are discrepancies between the computational model results and in vivo states that are difficult to identify using only computational approaches. An additional level of model curation can be achieved by integrating high-throughput experimental data with the framework of a computational model to put "content in context" [35]. This data integration can be

Table 1 Significant milestones for $\mathrm{T}$. fusca research and characterization

\begin{tabular}{llll}
\hline Year & Development & Group & Citation \\
\hline 1998 & Genus Constructed & Zhang et al. & Int. J Syst Bact. 1998 Apr \\
2002 & Physical Characterization & Kukolya et al. & Int. J Sys Evol Micr, 2002 Jul \\
2005 & Genome Sequenced & DOE & JGl Finished Genome, 2005 \\
$2004-2006$ & Plant biomass degradation study and analysis of & Wilson et al. & Chem Rec. 2004 \\
& enzymatic system & & Biochem. 2006 Nov \\
2007 & Sequence Annotation & Lykidis et al. & J. Bacteriol. 2007 Mar \\
2010 & First Genetic Modification & Deng \& Fong & Appl. Environ. Microbiol 2010 Apr \\
\hline
\end{tabular}




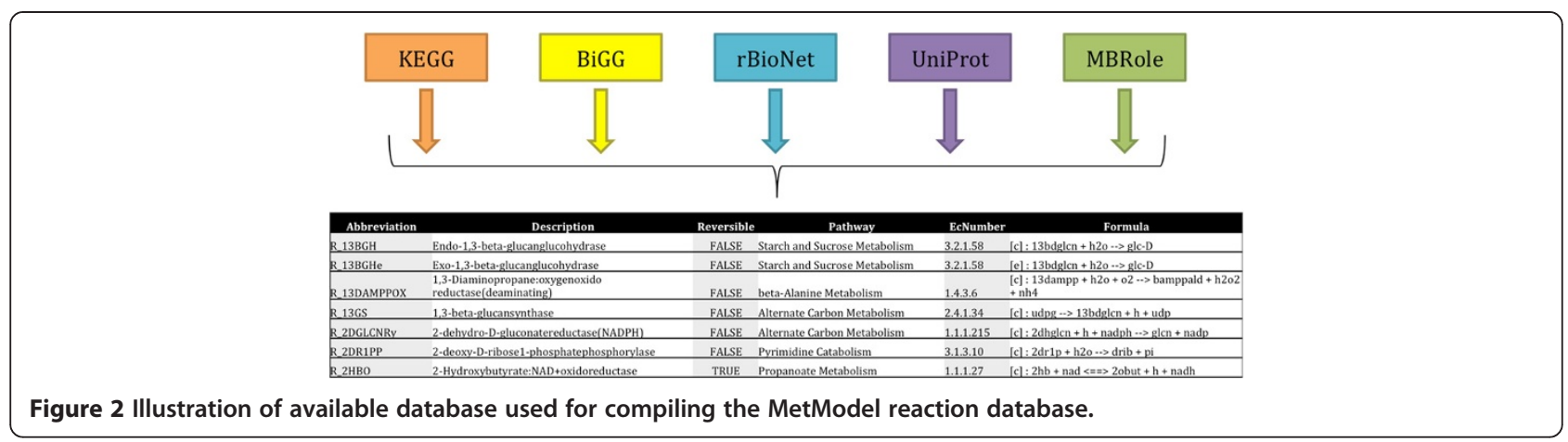

done using multi-scale high throughput experimental data such as transcriptomics, proteomics and metabolomics. This step reconciles in silico predictions with experimental results and thereby helps enhance the characterization of the cellular activity.

Genome-scale metabolic models have been created for many prokaryotic microbes and a variety of applications [23]. Several of these models have been able to incorporate experimental data to more closely match cellular processes. Once the model closely resembles a biological system, it can be optimized for defined objective function. This objective function may range from production of biomass to production of a chemical target. Following the in silico optimization of yields the computational design may eventually be replicated for applications in industry, therapeutics or health-related predictions.

In this study, three different approaches for generating constraint-based models were used and analyzed for their ability to accurately predict cellular growth when given an input substrate uptake rate. The three model versions were 1) designed using Model SEED [36], 2) an in-house semi-automated reconstruction based on organism specific annotation and reaction information from KEGG [21,22], and 3) a proteomics-based model from 2D proteomic experiment of $T$. fusca grown on cellobiose media. A comparative analysis between the different models was conducted to develop the most experimentally accurate model of $T$. fusca and to provide comparison of different model generation methods. Detailed analysis of metabolic function of T. fusca is also included to provide perspective on potential industrial applications (eg: in biofuel and natural product) for development of consolidated bioprocesses using T. fusca. Here we will be discussing the mevalonate and non-mevalonate pathways for terpenoid biosynthesis. These pathways are utilized for the production of isoprenoid precursor (isopentenyl pyrophosphate and dimethylalyl pyrophosphate) compounds that have applications in pharmaceutical, nutraceutical and perfume industries.

\section{Results and discussion}

$T$. fusca is known to directly use lignocellulosic biomass as a carbon substrate due to its well-studied cellulolytic system [1,37]. In laboratory conditions, $T$. fusca can grow on a variety of substrates including glucose, cellobiose, and microcrystalline cellulose. To match experimental work conducted as part of this study on cellobiose, it was necessary to add cellobiose degradation reactions to the draft model based upon the annotation and experimental evidence (EC 3.2.1.21, Tfu_0937). Three versions of a T. fusca model were created, as shown in Figure 3, using three different approaches to compare modelbuilding approaches and models were tested against experimental growth on cellobiose. In the process of model reconstruction, activity of terpenoid pathways was uncertain, but was experimentally verified using real-time PCR.

\section{Metabolic reconstruction: summary and model statistics}

Three different draft metabolic models were constructed for Thermobifida fusca. The three models varied based upon what was used as the starting point for generating the initial reaction list for the model (Model SEED, KEGG, proteomics data). In all cases, after initial generation of a reaction list, all reactions were associated with KEGG IDs to standardize comparisons between models.

Tfu_v1: The taxonomy number of $T$. fusca was used to generate a draft model from Model SEED (Tfu_v1) $[36,38]$. The Model SEED output was then converted to KEGG compound identifiers before running gap analysis and FBA. The Model SEED-derived Tfu_v1 model contained 1302 reactions, 1213 metabolites, and 618 EC numbers. Gap analysis added 146 reactions that primarily include a variety of exchange reactions (reactions that denote the direct uptake/secretion of the respective metabolite from or to the extra cellular media). When used with unconstrained carbon input flux (cellobiose uptake of $1000 \mathrm{mmoles} / \mathrm{gDW} / \mathrm{h}$ ) the Tfu_v1 model calculated a growth rate of 24.25 doublings/h. However, when the experimentally determined substrate uptake rate of cellobiose $(0.25 \mathrm{mmoles} / \mathrm{gDW} / \mathrm{h})$ was applied to this model it 


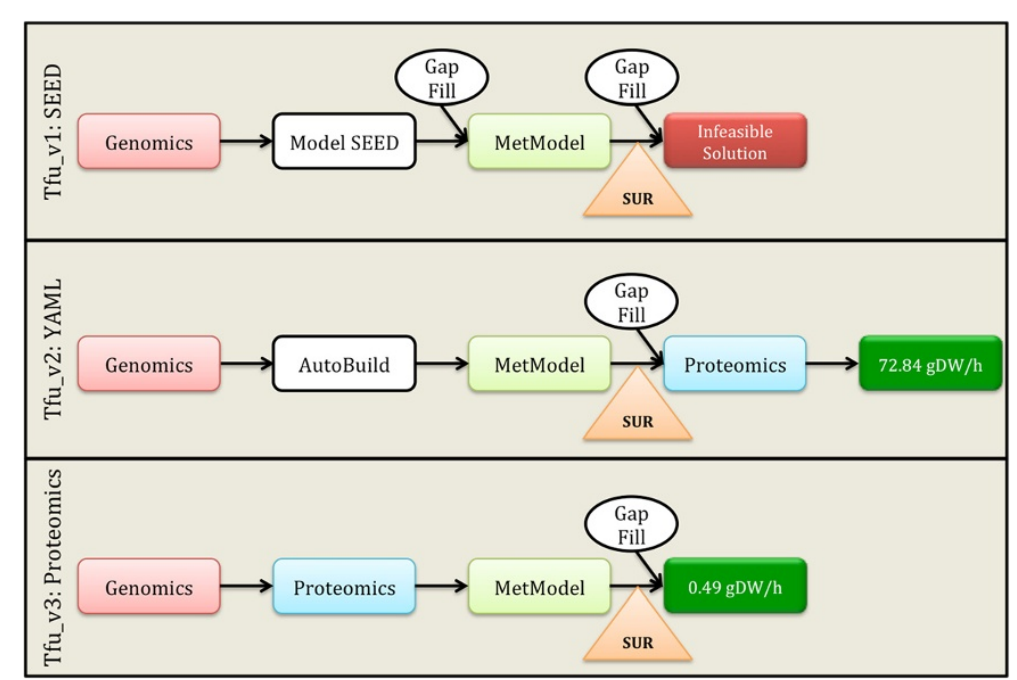

Figure 3 Flowchart of the model building step and data incorporation for all the three version of the model. Genomics and Proteomics are the high throughput datasets used for building the models. SUR - Substrate uptake rate for cellobiose as calculated experimentally was applied as a constraint. Red indicated no solution was obtained when running simulation of SEED model under experimental constraints. Values in the green boxes indicate the growth rates for Tvu_v2 and Tfu_v3.

failed to arrive at a viable solution (warning of infeasible solution). This infeasibility was crosschecked and verified by using the Model SEED FBA runs. The model failed to perform under any media formulations (glucose and cellobiose) attempted using the Model SEED simulation platform.

Tfu_v2: The second version of a $T$. fusca model (Tfu_v2) was created using an in-house semi-automated reconstruction method as defined in our past publications $[27,28]$. This model consists of 1002 reactions involving 584 EC numbers and accounting for 1105 metabolites. Eight out of 48 reactions added in the gap analysis were non-exchange reactions. Applying the experimentally determined constraint of substrate uptake rate for cellobiose as $0.25 \mathrm{mmoles} / \mathrm{gDW} / \mathrm{h}$, the optimal biomass growth was 72.84 doublings/h. This growth rate was compared to experimental growth rate of 0.43 doublings/h.

Tfu_v3: The third version of the model generated in this study (Tfu_v3) is based on proteomic experimental data generated by our group in collaboration with the Manitoba Centre for Proteomics \& Systems Biology, the University of Manitoba. The detailed protein characterization of T. fusca based on this data is beyond the scope of this paper and will be explained in future publications along with the other experimental data. This method of generating a model varied from Tfu_v1 and Tfu_v2 as the initial reaction list was populated from reactions associated with experimentally detected proteins, not informatics-based annotation. The Tfu_v3 model contains 975 reactions, 1382 metabolites, and 316 EC numbers (296 unique genes annotated in T. fusca). FBA simulations with an input constraint of a cellobiose uptake rate of 0.25 mmoles/ $\mathrm{gDW} / \mathrm{h}$ gave a predicted growth rate of 0.49 doublings $/ \mathrm{h}$ (for reference experimentally determined growth rate is 0.43 doublings $/ \mathrm{h}$ ). A comparison of the model contents (by reaction) is summarized in Figure 4 and the detailed model in all three versions is attached as Additional file 1 (Model.xls).

Due to the critical role of central metabolism to heterotrophic organisms, an analysis was conducted using all three model versions to study central metabolism. To study the individual pathways in carbohydrate metabolism and amino acid metabolism, each metabolic pathway was annotated using EC numbers recorded in KEGG. The summary table is shown in Table 2. Genes within a pathway were tabulated to evaluate the completeness of given pathway. At the first glance, all the pathways with a gene presence of $20 \%$ or above were counted as present/active.

\section{Tfu_v3: proteomics model reaction distribution}

Based upon the initial model contents and growth rate simulation results, the the Tfu_v3 appears to most closely predict experimental results. Thus, further detailed computational analyses of T. fusca are based on the Tfu_v3 model. The Tfu_v3 model is designated as iTfu296 (represents 296 annotated genes).

The proteomics-based model of T. fusca accounts for 216 amino acid reactions and 182 carbohydrate-related reactions as illustrated in Figure 5. When running an FBA simulation to maximize growth with an input cellobiose uptake of $0.25 \mathrm{mmoles} / \mathrm{gDW} / \mathrm{h}, 110$ reactions were 


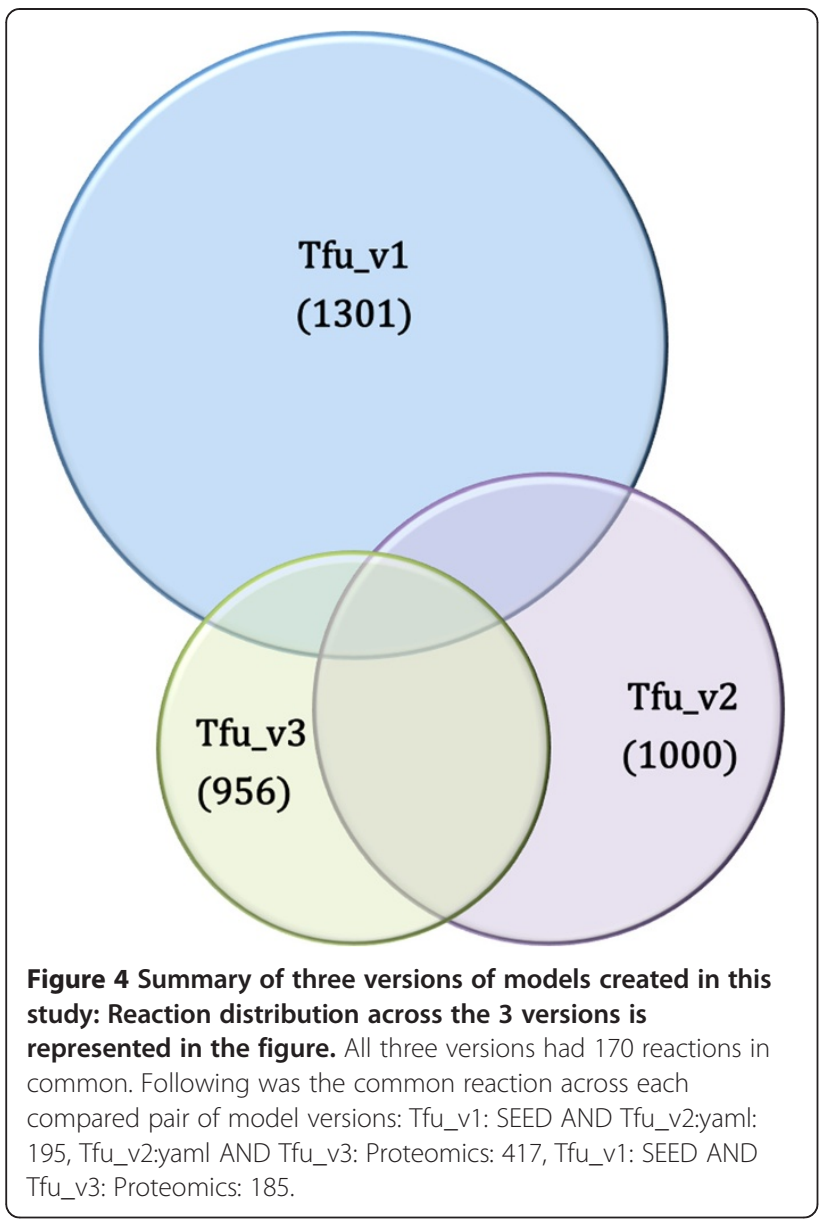

highly active as indicated by high calculated fluxes (flux values of $>100$ or $<-100$ ). (The range of flux through reactions was from 0 to 1000; with a threshold of 100 arbitrarilty selected for the purpose of analysis). Among these 110 reactions, the majority of the reactions were used in carbohydrate (37) and amino acid (34) metabolism (i.e. 60 unique reactions after eliminating overlaps between carbohydrates (37) and amino acid (34)).

In carbohydrate metabolism, the tricarboxylic acid cycle (TCA cycle) was complete and active. In the current study, we will be focusing on the seven compounds of TCA cycle that play crucial roles in carbon exchange between various pathways in the central metabolism. These intermediates include: pyruvate (KEGG compound C00022), acetoacetyl CoA (C00332), acetylyl CoA (C00024), alpha-ketoglutarate (C00026), succinyl CoA (C00091), fumarate (C00122) and oxaloacetate (C00036). Studying the reaction use and fluxes involving these compounds will help us understand the probable activity in amino acid biosynthesis and degradation pathways. To summarize these nodes in the current proteomics-based version of our model, 60 unique reactions (associated with
Table 2 Summary of individual pathway contrasts between three versions of model: The reference frames selected here is KEGG

\begin{tabular}{lllll}
\hline & $\begin{array}{l}\text { KEGG } \\
\text { reference } \\
\text { Reactions in } \\
\text { pathway }\end{array}$ & Tfu_v1 & Tfu_v2 & Tfu_v3 \\
& & & & \\
\hline
\end{tabular}

\section{Carbohydrates \\ Metabolism}

\begin{tabular}{|c|c|c|c|c|c|c|c|}
\hline Glycolysis/Gluconeogenesis & 45 & 28 & $62 \%$ & 22 & $49 \%$ & 15 & $33 \%$ \\
\hline Citrate Cycle (TCA Cycle) & 22 & 15 & $68 \%$ & 15 & $68 \%$ & 13 & $59 \%$ \\
\hline Pentose Phosphate Pathway & 40 & 20 & $50 \%$ & 15 & $38 \%$ & 13 & $33 \%$ \\
\hline $\begin{array}{l}\text { Pentose and Glucuronate } \\
\text { Intrconv. }\end{array}$ & 61 & 10 & $16 \%$ & 8 & $13 \%$ & 6 & $0 \%$ \\
\hline $\begin{array}{l}\text { Fructose and Mannose } \\
\text { Metabolism }\end{array}$ & 65 & 14 & $22 \%$ & 14 & $22 \%$ & 5 & (6 \\
\hline Galactose Metabolism & 38 & 12 & $32 \%$ & 13 & $34 \%$ & 8 & $21 \%$ \\
\hline $\begin{array}{l}\text { Starch and Sucrose } \\
\text { Metabolism }\end{array}$ & 74 & 14 & $19 \%$ & 14 & $19 \%$ & 12 & $16 \%$ \\
\hline $\begin{array}{l}\text { Amino Sugar and Nucleotide } \\
\text { Metabolism }\end{array}$ & 108 & 25 & $23 \%$ & 21 & $19 \%$ & 15 & \\
\hline Pyruvate Metabolism & 64 & 26 & $41 \%$ & 17 & $27 \%$ & 15 & $23 \%$ \\
\hline $\begin{array}{l}\text { Glyoxylate and Dicarboxylate } \\
\text { Metabolism }\end{array}$ & 66 & 12 & $18 \%$ & 11 & $17 \%$ & 7 & \\
\hline Propanoate Metabolism & 47 & 17 & $36 \%$ & 14 & $30 \%$ & 12 & 2 \\
\hline Butanoate Metabolism & 50 & 17 & $34 \%$ & 12 & $24 \%$ & 7 & $4 \%$ \\
\hline $\begin{array}{l}\text { C- } 5 \text { Branched Diabasic Acid } \\
\text { Metabolism }\end{array}$ & 18 & 3 & $17 \%$ & 3 & $17 \%$ & 1 & \\
\hline Inositol Phosphate & 43 & 4 & $9 \%$ & 5 & $12 \%$ & 4 & \\
\hline
\end{tabular}

\section{Amino Acid Metabolism}

Ala, Asp and Glu Metabolism 43

Gly, Ser and Thr Metabolism 63

Cys and Met Metabolism 66

Val, Leu and lle Degradation 35

Val, Leu and lle Biosynthesis 14

Lys Biosynthesis $\quad 30$

Lys Degradation $\quad 54$

Arg and Pro Metabolism 104

His Metabolism 37

Tyr Metabolism 66

Phe Metabolism $\quad 70$

Trp Metabolism 69

Phe, Tyr and Trp 37

Biosynthesis

$\begin{array}{llllll}21 & 49 \% & 19 & 44 \% & 18 & 42 \%\end{array}$

$\begin{array}{llllll}11 & 17 \% & 13 & 21 \% & 9 & 14 \%\end{array}$

$8 \quad 12 \% \quad 3 \quad 5 \% \quad 3 \quad 5 \%$

$\begin{array}{llllll}18 & 51 \% & 13 & 37 \% & 16 & 46 \%\end{array}$

$\begin{array}{llllll}10 & 71 \% & 10 & 71 \% & 7 & 50 \%\end{array}$

$\begin{array}{llllll}13 & 43 \% & 10 & 33 \% & 8 & 27 \%\end{array}$

$\begin{array}{lllllll}7 & 13 \% & 8 & 15 \% & 7 & 13 \%\end{array}$

$\begin{array}{llllll}32 & 31 \% & 26 & 25 \% & 22 & 21 \%\end{array}$

$\begin{array}{llllll}12 & 32 \% & 13 & 35 \% & 10 & 27 \%\end{array}$

$\begin{array}{llllll}11 & 17 \% & 8 & 12 \% & 9 & 14 \%\end{array}$

$\begin{array}{llllll}11 & 16 \% & 9 & 13 \% & 9 & 13 \%\end{array}$

$\begin{array}{llllll}14 & 20 \% & 14 & 20 \% & 9 & 13 \%\end{array}$

$\begin{array}{llllll}26 & 70 \% & 16 & 43 \% & 16 & 43 \%\end{array}$

Each of these pathways were individually screened to make a call for active or inactive by tracing through the List of EC numbers on the KEGG map. If majority (almost all) of the EC number continuously connecting the pathway were present then the pathways was marked active else inactive.

carbohydrates and amino acid metabolism) were found with some significant flux (i.e. flux was $>0.01$ units). Among these, 23 unique reactions correspond to amino 


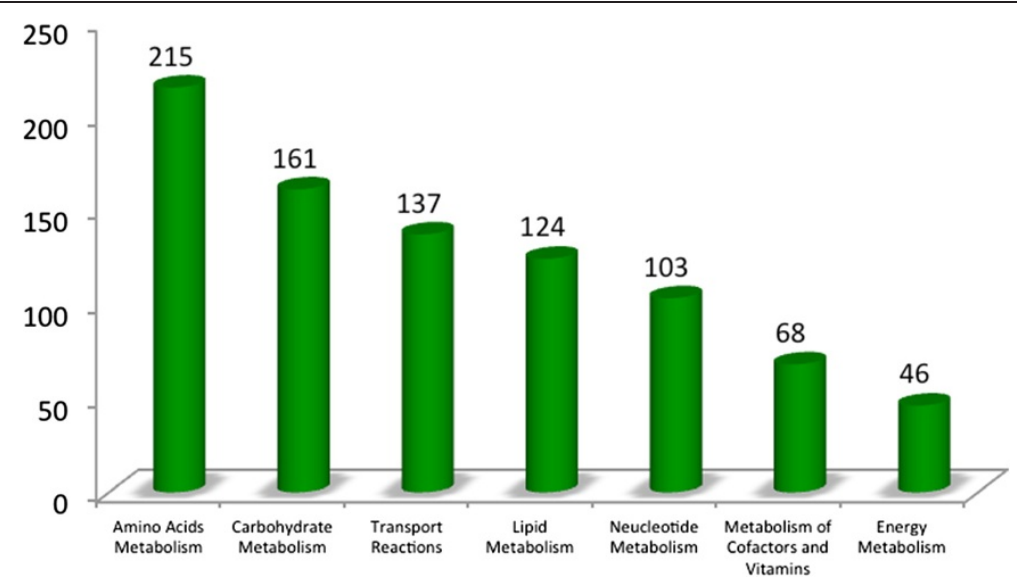

Figure 5 Major reaction distribution in the proteomics based model comprising total of 1002 reactions.

acid metabolism. This indicates the activity around the most of the amino acid - carbohydrate connection nodes as shown in Figure 6.

It was observed that in the carbohydrate-amino acid network connection the majority of reactions (11 out of 23) branch in and out of alpha - ketoglutarate. The acetyl CoA node was more centralized by connecting the amino acids and fatty acid pathways with 8 significantly high flux reactions. The pathway specific details are also illustrated in the Table 2. It was observed that when contrasted with the reactions specific to the biosynthesis or degradation of amino acids, the overall analysis suggests the presence of all but one amino acid subsystem, cysteine and methionine metabolism.

\section{Comparison of "model refinement with data" Vs "reconstruction from data"}

In the past, several attempts have been made to use computational metabolic models as a scaffold for experimental data integration [39-42]. Experimental data integration not only serves as a means of testing model predictions, but can also be used to help refine the model solution space to provide simulations that more closely match in vivo flux states. In this study, we used

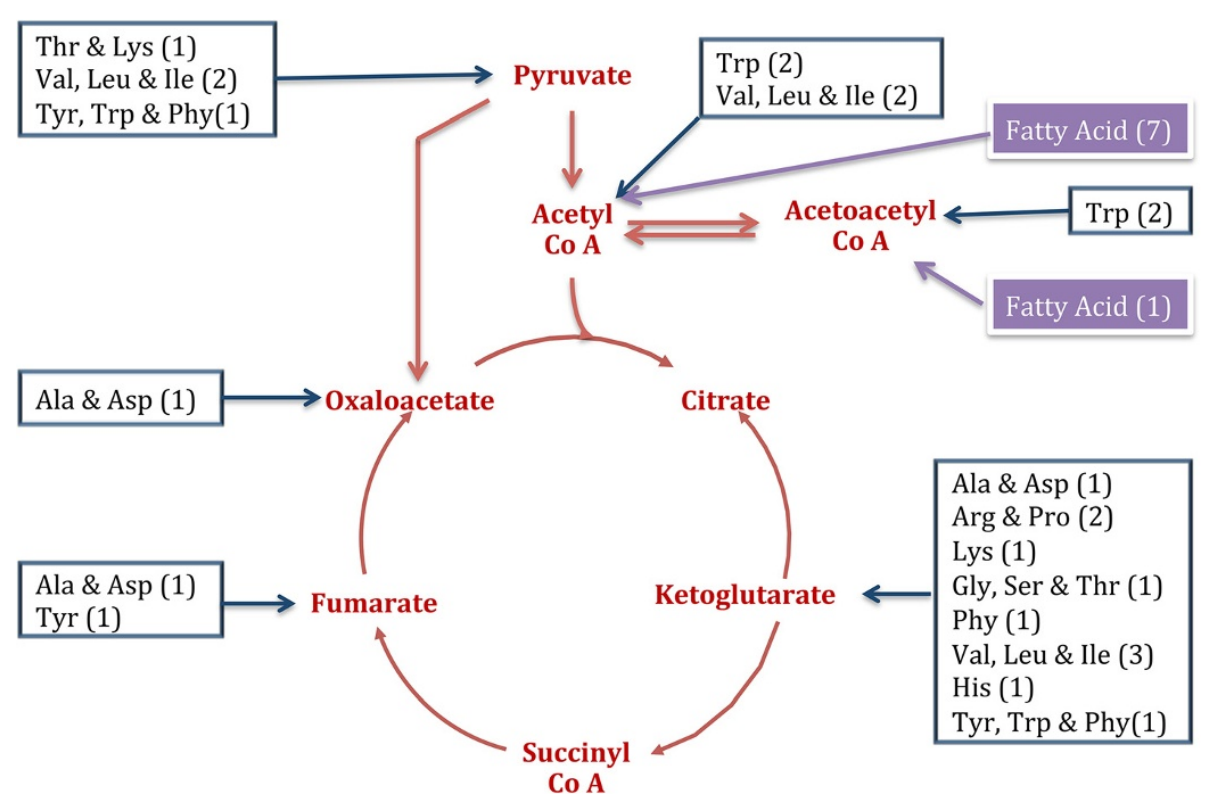

Figure 6 Summary of carbohydrate and amino acids connection nodes and number of active flux reaction within amino acid pathways. Standard three-letter amino acids code is used to denote the pathway names. The carbohydrate nodes of the central metabolism are highlighted as red and the branching out amino acid pathways are boxed in blue. The purple boxes denote the fatty acids connections from Acetyl Co A and Acetoacetyl Co A. The numbers of reactions for each pathway are represented in the parenthesis. 
two methods to combine metabolic models and experimental data to understand and characterize the metabolic network of T. fusca. For the first approach, an MILP algorithm (analogous to Shlomi et al. [42]) was used to integrate the proteomics dataset to the model Tfu_v2 (autobuild model). This algorithm aims at optimizing the agreement between the experimental data and the in silico model [42]. In this context, the experimental information is used to assign a present or absent call to re-channelize the flux distribution of the network as explained by Gowen et al. [43].

Parallel to this, a second approach originating directly from an experimental proteomic data set was used to generate an independent model, Tfu_v3. Currently existing methods for constraint-based model reconstruction primarily depend on the bioinformatic information such as genomics data, biochemical data and models of related microorganisms at the initial phase of model building. The approach taken for construction of Tfu_v3 relies on the in vivo experimental evidence of the proteins as the starting point of model building. While these two methods of model construction used the same bioinformatics and experimental data, subtle differences in the order of process steps and algorithms, as shown in Figure 5, used to construct the two models resulted in vastly different functional consequences.

Besides this, another very interesting and significant difference between Tfu_v2 and Tfu_v3 was observed in the function of the TCA cycle. The reaction using pyruvate to make oxaloacetate was not found in the autobuilt Tfu_v2 version whereas the proteomics version clearly shows its presence (EC 6.4.1.1, Gene ID: Tfu_2557, Tfu_1530, Tfu_0947, Tfu_1228). In addition, most of the amino acid pathways were fully or partially incomplete in Tfu_v2. Thus, the Tfu_v2 model had artificially high fluxes through transport reactions to uptake external nutrients to satisfy simulation requirements for optimal biomass production. The Tfu_v3 model showed most of the pathways significantly complete except cysteine and methionine metabolism. Besides cysteine and methionine metabolism, phenylalanine metabolism was sparsely populated, but active flux through the reaction involved in the interconversion of phenylalanine to phenylpyruvate suggested making a present call for the entire phenylalanine pathway. These functional differences indicate some of the potential danger associated with over-reliance on genome annotation (that may contain numerous errors especially in under-characterized organisms).

\section{Applicability of the model: biofuels and pharmaceutical precursors}

The subsystem-based analysis of central metabolism and experimental foundation suggests the closer association of Tfu_v3 to the in vivo biochemical system of T. fusca.
While the Tfu_v3 model is the most accurate of the three models constructed in this study, there are numerous areas of metabolism that are not well-characterized. For comparison, even the most update model of $E$. coli has an account of only for $30 \%$ of the gene products in the model [44]. Likewise, this model being the first ever T. fusca metabolic network also opens huge scope of pathway-focused review and improvement. Once completely functional these models provide a ground for hypothesizing a target for the further study.

T. fusca is a potentially interesting organism for biochemical production of sustainable fuels or industrial chemicals. In these areas, two pathways of particular interest are butanol and secondary metabolite biosynthesis (e.g. terpenoids). The Tfu_v2 (ModelSEED autobuilt) model incorporates most of the reactions present in the butanoate metabolism however, no active flux was observed through most of them. Tfu_v3 based on experimental dataset confirms approximately $50 \%$ of these reactions but also predicts no active flux through these pathways. Thus, while production of butanol through butanoate metabolism appears possible in terms of biochemical capabilities, it remains to be explored and demonstrated experimentally. For comparison purposes, in 2011 a mutant strain T. fusca B6 was designed and constructed with heterologous expression of a bifunctional alcohol dehydrogenase (adhE2) for production of 1-propanol [12]. Engineered production of 1-propanol in T. fusca provides first-step experimental evidence that $T$. fusca may be usable for the production of fuels directly from lignocellulosic raw materials.

One consideration for FBA simulations of both butanol/butanoate and terpenoids is that these are secondary pathways and utilization of these pathways are not explicitly included in the biomass objective for growth maximization simulations. Thus, it is not necessarily surprising that growth simulations with no genetic designs incorporated may not show flux through secondary pathways. However, due to the potential for secondary metabolism in actinomycetes, we considered studying the terpenoid backbone (TBB) pathway in more detail.

With the aim of characterization, 16 reactions for the TBB pathway were added to the model to computationally test the feasibility of flux through the TBB pathway when optimizing for the biomass production. All the reactions of the mevalonate pathway except R001123 had an active flux of approximately $0.53 \mathrm{mmoles} / \mathrm{gDW} / \mathrm{h}$. The reaction R01123 was responsible for isomerization reaction between isopentenyl diphosphate (IPP or C00129) and dimethylallyl pyrophosphate (DMAPP or C00235). The positive flux through the reaction (R01121) lead to the formation of IPP, as shown in Figure 7, and provided computational support that $T$. fusca may possess the capability to divert the flux through the mevalonate pathway 


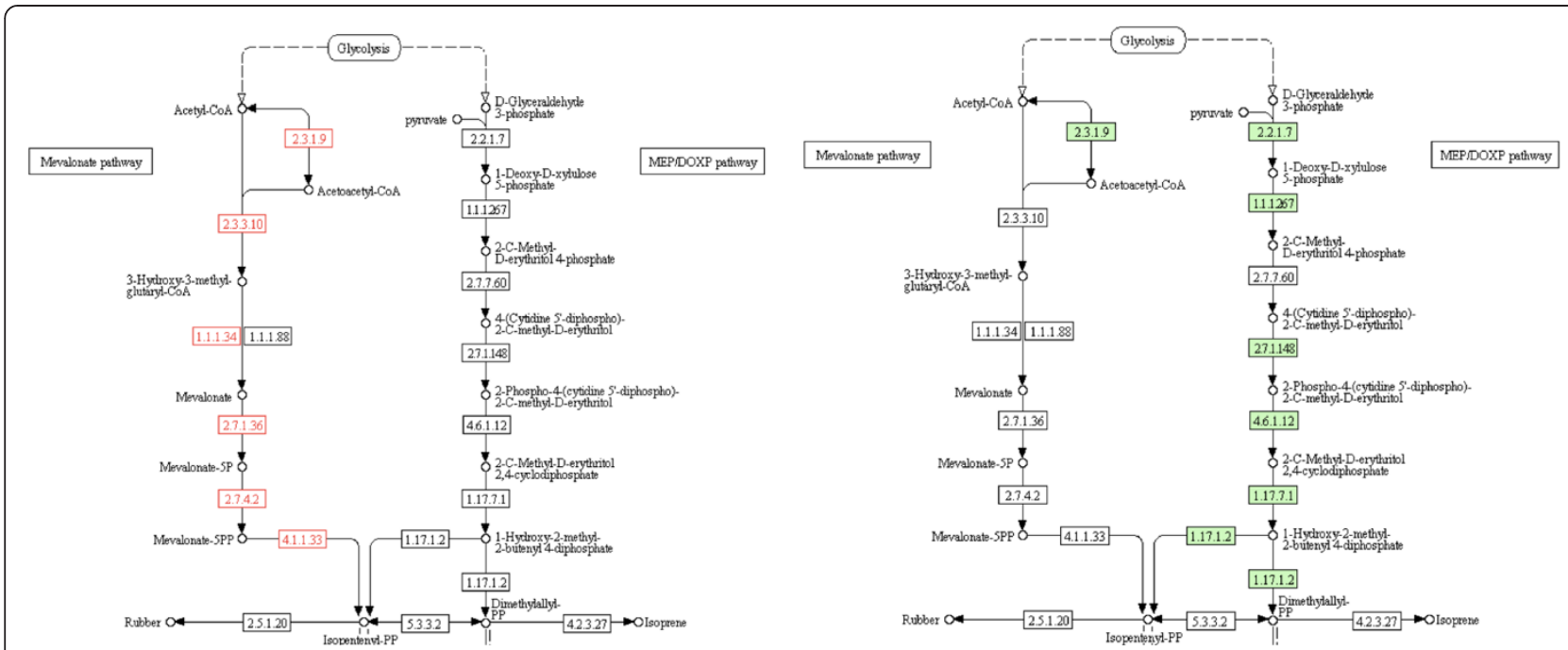

Figure 7 TBB as from flux analysis through Mevalonate Pathway (left) and expression analysis of Non mevalonate pathway (right).

reactions. This data was used as a clue to conduct followup experiments to verify the active in vivo expression of these pathways.

\section{Experimental validation: expression analysis TBB pathway genes}

Different bacteria have been found to produce terpenoids by either the mevalonate pathway or the nonmevalonate pathway (DXP pathway). While computational simulations demonstrated the feasibility of an active mevalonate pathway, no clear evidence existed for either the mevalonate or the DXP pathways. Given the shorter length of the DXP pathway, initial experimental testing focused on the seven genes of the DXP pathway (Figure 8).

Real-time PCR was used to measure mRNA transcript levels for the genes in the DXP pathway. The details of T. fusca genes studied and tested are listed in Table 3 along with their relative expression in Figure 9. All but one of the required genes were found to be expressed in the strain. The missing enzyme was 2-C-methyl-D-erythritol 4-phosphate cytidylyltransferase (EC: 2.7.7.60). This suggests the presence of terpenoid backbone biosynthesis via the DXP pathway in T. fusca.

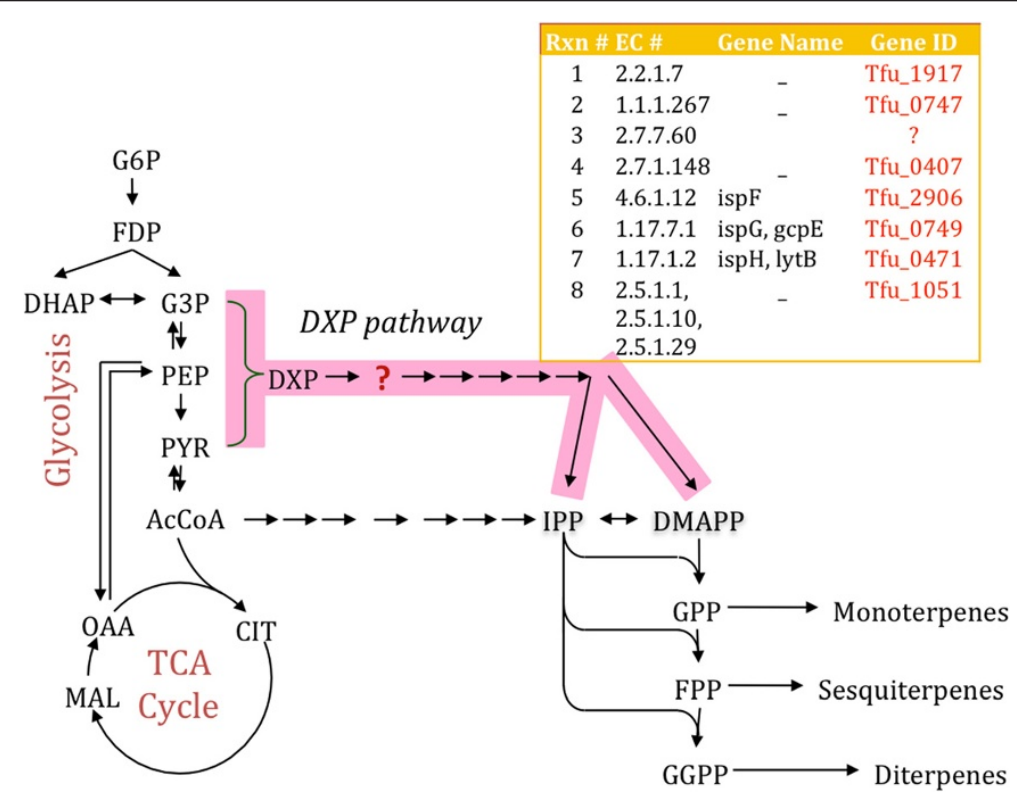

Figure 8 TBB through the non-mevalonate pathway and gene annotation in T. fusca. 
Table $3 \mathrm{~T}$. fusca genes associated with the DXP pathway for terpenoid biosynthesis

\begin{tabular}{llllll}
\hline Locus & NCBI_GenelD & Strand & $\begin{array}{l}\text { Start } \\
\text { Pos }\end{array}$ & $\begin{array}{l}\text { End } \\
\text { Pos }\end{array}$ & $\begin{array}{l}\text { NT } \\
\text { seq }\end{array}$ \\
\hline Tfu_1917 & 3580825 & Complementary & 2242018 & 2243931 & 1914 \\
Tfu_0747 & 3578952 & & 880259 & 881473 & 1215 \\
Tfu_0407 & 35800006 & & 458401 & 459324 & 924 \\
Tfu_2906 & 3581392 & Complementary & 3421460 & 3421924 & 465 \\
Tfu_0749 & 3578954 & & 883104 & 884261 & 1158 \\
Tfu_0471 & 3579613 & & 532475 & 533476 & 1002 \\
Tfu_3076 & 3580076 & & 3598495 & 3599487 & 993 \\
\hline
\end{tabular}

\section{Conclusions}

Three different methodologies were applied to create metabolic reconstructions for T. fusca. The proteomicsbased model (Tfu_v3) named $i$ Tfu296 was found to mimic the biological growth conditions most closely. It was observed that when cellobiose uptake rate was constraint as $0.25 \mathrm{mmoles} / \mathrm{gDW} / \mathrm{h}$ gave the growth rate of 0.49 doublings/h. This was comparable to the experimental growth rate of 0.43 doublings $/ \mathrm{h}$. This model was built using a novel scheme for model reconstruction based on high throughput proteomic data at the initial model building phase.

Genomic datasets are the most standard high throughput data currently available, but it is always a concern to what extent the genomics information is really transcribed and translated into the functional role inside the cell. Out of 3195 genes annotated in the T. fusca genome published in 2005, only 3117 translated into proteincoding genes and only 1757 were associated with predicted functions $[11,45]$. This discrepancy between genomic and proteomic information may be due to environmental or evolutionary selection processes. Accurate proteomic data provides more information about the functional activities of the cell, as confirmed by the fact that our proteomics-based model agreed with experimental observations better than models built based on genomic information alone.

With the advent of standard genomics information, genome-scale metabolic models have become a widely used approach to gain a systems level understanding of metabolic processes and function [31]. Every organismspecific model that is built needs to pass through multiple levels of curation and validation based on genome annotation, experimental evidence and (or) biochemical literature study. Knowing that omics-data can be used to help identify in vivo function, we applied an approach model building based on high throughput proteomic data. These models contain the scope of integration of genomics, transcriptomics, proteomics, metabolomics and phenomics data. For the current study, proteomics data has been used to establish a significantly reliable starting point for the metabolic model reconstruction. This version of model Tfu_v3 is based on functional building blocks that are more closely associated with the phenotypic characteristics when compared to genomics data in the hierarchy.

In a larger context, the modeling approach aims at establishing links between the molecular and cellular functions. However, it is still hard to find complete agreement between the "biology - biochemistry" and "network models omics data". It is reported that the most updated $E$. coli [44] model only associates to $30 \%$ of gene products in the model and $1 / 3$ of the gene products are not functionally annotated [46]. Even with this limitation, model-based systems analysis can be useful for developing hypothesis and target for the focused study. In this case, we hypothesize the presence of an active terpenoid backbone pathway, which will be the focus of our follow up studies using experimental and analytical methods.

Nevertheless, this promising approach suffers with a major limitation to date - a lack of a standardized reaction database to build and analyze metabolic network. Due to

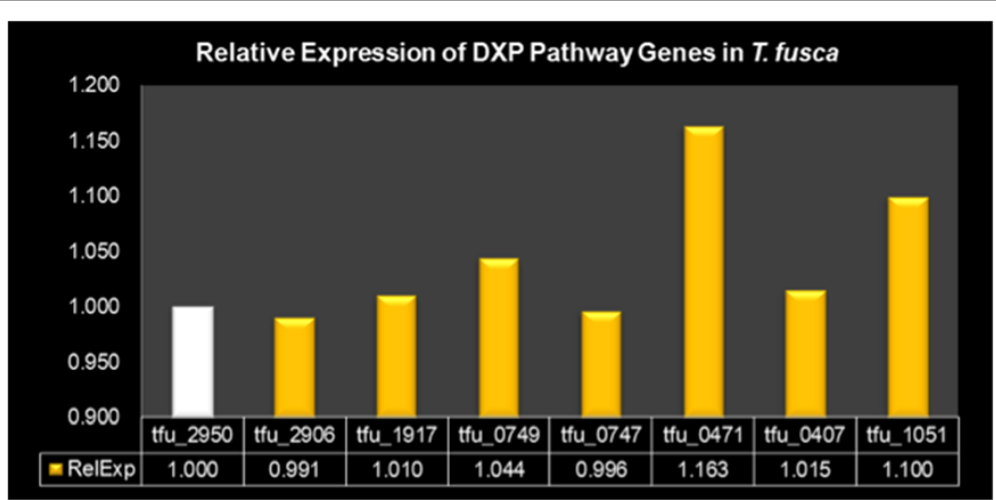

Figure 9 Relative expression of terpenoid biosynthetic genes associated with the DXP pathway in T. fusca. Tfu_2950 is a housekeeping gene with constitutive expression in T. fusca. 


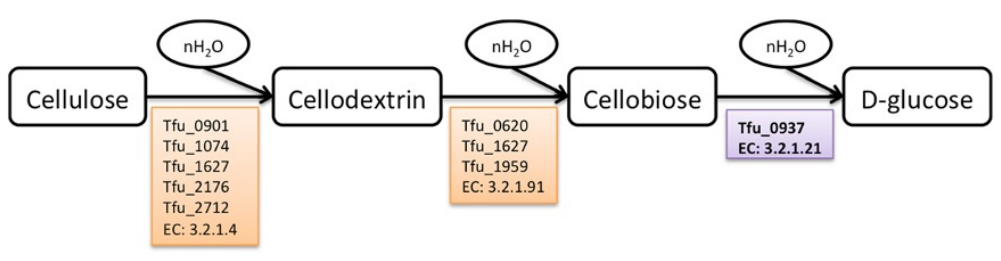

Figure 10 Cellulose degradation reaction in presence of water: The reaction to convert cellobiose as per the experimental dataset Tfu_0937, EC 3.2.1.21 was expressed and thus the associated reaction was added to the model.

inconsistency in the labeling of the metabolites (eg: citrate is almost chemically equivalent to citric acid; 2-hydroxy1,2,3-propanetricarboxylic acid; 2-hydroxytricarballylic acid and have the same compound identifier on KEGG) it is difficult to assemble a non-redundant reaction database with standard nomenclature. Current systems biology experts are in the quest of cleaning and populating available database with minimal redundancy in the hope of exhaustive coverage of cellular biochemical reactions. Some of the examples are MetRxn and MetaCyc.

Computational analysis demonstrated the theoretical feasibility of producing terpenoids in T. fusca, however, no existing experimental evidence had previously supported or demonstrated this capability. Analysis of mRNA transcripts showed in vivo activity of the DXP pathway in $T$. fusca providing evidence that $T$. fusca may be capable of direct cellulose-to-terpenoid biosynthesis. Isoprene is the monomeric unit for the huge family of terpenoids, thus hold importance in pharmaceutical industry, perfumes, incense, flavoring, spices, and varnishes.

T. fusca was found to be a biofuel producing strain after the genetic modification protocol for this strain was established by Deng \& Fong. With this systems level characterization of secondary metabolites, it can be suggested as a highly useful, robust and inexpensive strain for industrial application. However, this opens an arena for the scale up and optimization study to successfully launch this strain in industrially significant microbes.

\section{Methods}

\section{Culture conditions}

Thermobifida fusca ATCC BAA-629 was grown in Hagerdahl medium containing $1.0 \%$ cellobiose. Experiments were conducted in Erlenmeyer flasks where $50 \mathrm{~mL}$ precultures of $T$. fusca $\mathrm{YX}$ were grown at $55^{\circ} \mathrm{C}$ and $250 \mathrm{rpm}$ for 24 hours in a $500 \mathrm{~mL}$ Erlenmeyer flask. Growth

Table 4 Reactions associated with Terpenoids backbone biosynthesis pathway that were added to the model Tfu_v3 for the simulation of Secondary metabolites pathway central hub

\begin{tabular}{|c|c|c|c|}
\hline Rxn ID & Enzyme & EC Number & Equation \\
\hline \multicolumn{4}{|c|}{ \#Mevalonate pathway } \\
\hline R00238 & acetyl-CoA C-acetyltransferase & 2.3.1.9 & {$[c]:$ C00024 - > C00332 } \\
\hline R01978 & hydroxymethylglutaryl-CoA synthase & 2.3.3.10 & {$[c]: C 00332+C 00024->C 00356$} \\
\hline R02082 & 3-hydroxy-3-methylglutaryl-CoA reductase & 1.1.1.34 & {$[c]:$ C00356 - > C00418 } \\
\hline $\mathrm{R} 02245$ & mevalonate kinase & 2.7.1.36 & {$[c]:$ C00418 - > C01107 } \\
\hline $\mathrm{R} 03245$ & phosphomevalonate kinase & 2.7.4.2 & {$[c]:$ C01107 - > C01143 } \\
\hline R01121 & diphosphomevalonate decarboxylase & 4.1 .1 .33 & {$[c]:$ C01143 - > C00129 } \\
\hline R01123 & isopentenyl-diphosphate delta-isomerase & 5.3.3.2 & {$[c]:$ C00129 - > C00235 } \\
\hline \multicolumn{4}{|c|}{ \#Non mevalonate pathway } \\
\hline R05636 & DOXP synthase (Dxs) & 2.2.1.7 & [c]: C00118+C00022 - > C11437 \\
\hline R05688 & DOXP reductase (Dxr) & 1.1 .1 .267 & {$[C]:$ C11437 - > C11434 } \\
\hline R05633 & MEP synthase (IspD) & 2.7 .7 .60 & {$[c]:$ C11434 - > C11435 } \\
\hline R05634 & CDP-ME kinase (IspE) & 2.7.1.148 & {$[c]:$ C11435 - > C11436 } \\
\hline R05637 & CDP-MEP synthase (IspF) & 4.6 .1 .12 & {$[C]:$ C11436 - > C11453 } \\
\hline R08689 & HMB-PP synthase (IspG) & 1.17.4.3 & {$[c]:$ C11453 - > C11811 } \\
\hline R05884 & HMB-PP reductase (IspH) & 1.17.7.1 & {$[c]:$ C11811 - > C00129 } \\
\hline R08209 & HMB-PP reductase (IspH) & 1.17.1.2 & {$[c]:$ C11811 - > C00129 } \\
\hline R01123 & IPP delta-isomerase & 5.3.3.2 & [c]: C00129 - > C00235 \\
\hline
\end{tabular}


cultures for testing were inoculated using $5 \%$ of the preculture and grown at $55^{\circ} \mathrm{C}$ and $250 \mathrm{rpm}$ for $42-48$ hours.

\section{Metabolic network reconstruction}

Overall model construction steps illustrated in Figure 3 are as follows:

Tfu_v1: SEED. The autobuilt draft model was made in Model SEED [36,38] and used as the draft network.

The *xml file downloaded was converted into in-house MetModel format to run the FBA simulations with MetModel software.

Tfu_v2: yaml. The *.yaml autobuilt drafts were created by our group in 2008 using the then available organism specific annotations and reaction database from KEGG. $[27,29,43]$.

Tfu_v3: Proteomics. The draft model was constructed using the output of a 2-dimensional LC-MS analysis performed at the Manitoba Centre for Proteomics \& Systems Biology (University of Manitoba, Winnipeg, Canada). Cellobiose grown T. fusca sample was subjected to FASP lysis/digestion procedure (J.R. Wisnewski et al. Nature Methods 2009. 6(5). 359-362) followed by 2D-HPLC-MS/MS acquisition using TripleTOF 5600 mass spectrometer (ABSciex, Mississauga, ON) [47]. Thirteen pairwise-concatenated fractions in the first dimension were analyzed over a 1-hour HPLC-MS/MS session, each. This collection yielded 276,129 MS/MS spectra that were interrogated using an in-house GPU-based search engine [48] and yielded identification of 126,471 peptides (16,598 non-redundant) spanning 2101 proteins. This represents approximately $68 \%$ total proteomic coverage. Protein identification expectation values were computed using a Bayes' theorem application of its member peptide expectation values, following the design by Beavis and Fenyo for X!tandem [49]. Over 1700 proteins have expectation values of $\log (\mathrm{e})<-10$ (a one in ten-billion probability of random miss-assignment).

Open source T. fusca (Taxon identifier: 269800) database KEGG [21,22], IMG [45] and UniProt [25] was used to map the gene identifiers to the EC numbers which was then looked up as the search identifier in the reaction database compiled in-house. Further, manual curation was done using the published biochemical literature specifically for cellobiose utilization as explained in Figure 10.

\section{Linear programming for flux balance analysis}

In-house python scripts were used to run the FBA simulations using the linear programming algorithm as shown in the introduction.

\section{Objective function: biomass equation}

This draft model aims at the growth optimization and the best estimates used in metabolic modeling scenario are biomass equation, which was designed by slightly manipulating the biomass equations from closely related species and available published information about $T$. fusca growth conditions [50]. The box below shows the biomass equation used for the simulation of all the version of the model. The details of the compounds identifiers are listed in the Additional file 2.

$$
\begin{aligned}
& \text { BIOMASS EQUATION } \\
& 34.7964805 \text { C00001 + 40.1701382 C00002 } \\
& +0.00780937 \mathrm{C} 00003+0.00780937 \mathrm{C} 00006 \\
& +0.00780937 \mathrm{C} 00010+0.00780937 \mathrm{C} 00016 \\
& +0.00780937 \mathrm{C} 00018+0.00780937 \mathrm{C} 00019 \\
& +0.25601316 \mathrm{C} 00025+0.00780937 \text { C00034 } \\
& +0.59580299 \text { C00037 }+0.00780937 \text { C00038 } \\
& +0.50005823 \text { C00041 }+0.20910125 \text { C00044 } \\
& +0.33354560 \text { C00047 + 0.23467873 C00049 } \\
& +0.00780931 \text { C00059 + 0.28827498 C00062 } \\
& +0.12987656 \mathrm{C} 00063+0.25601316 \mathrm{C} 00064 \\
& +0.20970184 \text { C00716 + 0.00780937 C00070 } \\
& +0.14934101 \mathrm{C} 00073+0.14026667 \mathrm{C} 00075 \\
& +0.00780937 \mathrm{C} 00076+0.05515731 \mathrm{C} 00078 \\
& +0.18056213 \text { C00079 + 0.13425080 C00082 } \\
& +0.08898018 \text { C00097 + 0.00780937 C00698 } \\
& +0.43865670 \text { C00123 + 0.01174687 C00131 } \\
& +0.09262265 \text { C00135 + 0.21542571 C00148 } \\
& +0.23467873 \text { C00152 + 0.00780937 C00175 } \\
& +0.41159840 \mathrm{C} 00183+0.24664683 \mathrm{C} 00188 \\
& +0.00780937 \text { C00238 + 0.00780937 C00255 } \\
& +0.01174687 \text { C00286 + 0.00780937 C00305 } \\
& +0.28255111 \text { C00407 + 0.01174687 C00458 } \\
& +0.01174687 \text { C00459 + 0.09247649 C04574 } \\
& +0.00780937 \text { C14818 + 0.00780937 C14819 } \\
& +0.00780937 \text { C00229 - - > 40.0000000 C00008 } \\
& +39.9921906 \text { C00009 + 0.60239528 C00013 } \\
& +40.0000000 \text { C00080 + 0.00780937 C03688 }
\end{aligned}
$$

\section{Gap analysis and model comparison}

The draft model consists of the list of reactions however there are patches in the network that obstruct continuous flow of flux through the pathway. These links are filled in by using the reaction databank and suggesting the list of reactions required to complete the network. FBA-GAP is used to suggest the connection nodes/reactions that are missing [30]. The use and application of 
this framework have been described in past by Roberts et al., Gowen et al. and Vanee et al. $[27,29,43]$. FBA-GAP takes a draft model and biomass reaction and uses distances in the reaction network and mathematical optimization to produce a list of metabolites that are necessary for biomass production but cannot be produced or consumed by the cell. Reactions producing and consuming this list of metabolites are obtained from a reference database. These potentially gap-filling reactions were manually checked for relevant evidence such as associated proteins/enzymes characterized or genome annotations,. On detecting the specific evidence these reaction additions to the model wer accepted. The process is repeated until a positive biomass flux value is obtained. In this way, only high-confidence reactions are used to complete the reconstruction.

\section{Data integrations and model validation for Tfu_v2}

The mixed integer linear programming algorithm (MILP) published in 2008 by Shlomi et al. [42] was used for integration of proteomics data to the Tfu_v2 version of model. This algorithm was re-written in python by Gowen et al. [43] to include in our MetModel package.

\section{Characterization of TBB pathway}

Metabolic model $i$ Tfu296 or (Tfu_v3) was used to enlist the already existing secondary metabolites production pathway based reactions (characterized by EC numbers) and metabolites (characterized by KEGG compound IDs). In addition, besides studying the complete secondary metabolite network a focused study on terpenoids backbone biosynthesis (TBB) pathway using mevalonate and non-mevalonate pathway was performed using KEGG (map00900). The reactions database of these pathways was created using the information from KEGG as shown in the Table 4 below.

\section{Expression analysis of TBB pathway genes}

T. fusca strain YX grown on Cellobiose media to till the early $\log$ phase with dry cell weight of $2.005 \mathrm{mg} / \mathrm{mL}$ was used for isolation of RNA using the Qiagen RNA Protect and Qiagen RNAeasy kit. The total RNA was sent to Nucleic Acid Research Facility (Virginia Commonwealth University) for RT-PCR. Tfu_2950 was selected as housekeeping gene to measure the relative expression levels.

\section{Additional files}

Additional file 1: Three_Versions_of_Model.xlxs. xlxs file with three spreadsheets illustrative the reaction flux in three version of the model.

Additional file 2: Biomass Equation Compound Identifiers.xlxs. x|xs file with the list of reactants and products on the biomass equation and their description from Kegg database.
Competing interests

The authors declare that they have no competing interests.

\section{Authors' contributions}

NV constructed, analyzed and compared the three versions of model. NV and JPB ran the model simulation using MetModel the scripts. NV, VS, DS, OK and JAW worked on proteomics experiments to generate, pre-process and analyze proteomics data. NV integrated the experimental dataset with the model and completed model validation with the programming support from JPB. YD worked on growth curve experimental analysis, biomass equation and first draft of the version one of model. SSF conceived of the study. All authors read and approved the final manuscript.

\section{Acknowledgements}

This work was partially supported by Genome Canada (MGCB ${ }^{2}$ project). The funding agency was not involved in any aspect of the study (design, execution, writing, publication submission).

\section{Author details}

${ }^{1}$ Virginia Commonwealth University, Richmond, USA. ${ }^{2}$ University of Manitoba, Winnipeg, Canada. ${ }^{3}$ Kansas State University, Olathe, USA.

Received: 24 April 2014 Accepted: 14 July 2014

Published: 13 August 2014

\section{References}

1. Wilson DB: Studies of Thermobifida fusca plant cell wall degrading enzymes. Chem Rec 2004, 4:72-82.

2. Ghangas GS, Wilson DB: Cloning of the thermomonospora fusca endoglucanase E2 Gene in streptomyces lividans: affinity purification and functional domains of the cloned gene product. Appl Environ Microbiol 1988, 54:2521-2526.

3. Irwin DC, Zhang S, Wilson DB: Cloning, expression and characterization of a family 48 exocellulase, Cel48A, from Thermobifida fusca. Eur J Biochem 2000, 267:4988-4997.

4. Spiridonov NA, Wilson DB: Regulation of biosynthesis of individual cellulases in Thermomonospora fusca. J Bacteriol 1998, 180:3529-3532.

5. Kukolya J, Nagy I, Laday M, Toth E, Oravecz O, Marialigeti K, Hornok L: Thermobifida cellulolytica sp. nov., a novel lignocellulose-decomposing actinomycete. Int J Syst Evol Microbiol 2002, 52:1193-1199.

6. Lee J, Postmaster A, Soon HP, Keast D, Carson KC: Siderophore production by actinomycetes isolates from two soil sites in Western Australia. Biometals 2012, 25:285-296.

7. Takahashi S, Toyoda A, Sekiyama Y, Takagi H, Nogawa T, Uramoto M, Suzuki R, Koshino H, Kumano T, Panthee S, Dairi T, Ishikawa J, Ikeda H, Sakaki Y, Osada H: Reveromycin A biosynthesis uses RevG and RevJ for stereospecific spiroacetal formation. Nat Chem Biol 2011, 7:461-468.

8. Niraula NP, Kim SH, Sohng JK, Kim ES: Biotechnological doxorubicin production: pathway and regulation engineering of strains for enhanced production. Appl Microbiol Biotechnol 2010, 87:1187-1194.

9. Cane $\mathrm{DE}$, Ikeda $\mathrm{H}$ : Exploration and mining of the bacterial terpenome. Acc Chem Res 2012, 45:463-472.

10. Citron CA, Gleitzmann J, Laurenzano G, Pukall R, Dickschat JS: Terpenoids are widespread in actinomycetes: a correlation of secondary metabolism and genome data. Chembiochem 2012, 13:202-214.

11. Lykidis A, Mavromatis K, Ivanova N, Anderson I, Land M, DiBartolo G, Martinez M, Lapidus A, Lucas S, Copeland A, Richardson P, Wilson DB, Kyrpides N: Genome sequence and analysis of the soil cellulolytic actinomycete Thermobifida fusca YX. J Bacteriol 2007, 189:2477-2486.

12. Deng $Y$, Fong SS: Metabolic engineering of Thermobifida fusca for direct aerobic bioconversion of untreated lignocellulosic biomass to 1-propanol. Metab Eng 2011, 13:570-577.

13. Carere CR, Sparling R, Cicek N, Levin DB: Third generation biofuels via direct cellulose fermentation. Int J Mol Sci 2008, 9:1342-1360.

14. Edwards JS, Covert M, Palsson B: Metabolic modelling of microbes: the flux-balance approach. Environ Microbiol 2002, 4:133-140.

15. Orth JD, Thiele I, Palsson BO: What is flux balance analysis? Nat Biotechnol 2010, 28:245-248.

16. Varma A, Palsson BO: Metabolic flux balancing: basic concepts, scientific and practical use. Nat Biotechnol 1994, 12:994-998. 
17. Segre D, Vitkup D, Church GM: Analysis of optimality in natural and perturbed metabolic networks. Proc Natl Acad Sci U S A 2002, 99:15112-15117.

18. Shlomi T, Berkman O, Ruppin E: Regulatory on/off minimization of metabolic flux changes after genetic perturbations. Proc Natl Acad Sci U S A 2005, 102:7695-7700.

19. Rapoport TA, Heinrich R, Jacobasch G, Rapoport S: A linear steady-state treatment of enzymatic chains. A mathematical model of glycolysis of human erythrocytes. Eur J Biochem 1974, 42:107-120.

20. Durot M, Bourguignon P-Y, Schachter V: Genome-scale models of bacterial metabolism: reconstruction and applications. FEMS Microbiol Rev 2009, 33:164-190.

21. Kanehisa M, Goto S: KEGG: kyoto encyclopedia of genes and genomes. Nucleic Acids Res 2000, 28:27-30.

22. Kanehisa M, Goto S, Hattori M, Aoki-Kinoshita KF, Itoh M, Kawashima S, Katayama T, Araki M, Hirakawa M: From genomics to chemical genomics: new developments in KEGG. Nucleic Acids Res 2006, 34:D354-D357.

23. Schellenberger J, Park JO, Conrad TC, Palsson BØ: BiGG: a Biochemical Genetic and Genomic knowledgebase of large scale metabolic reconstructions. BMC Bioinformatics 2010, 11:213.

24. Thorleifsson SG, Thiele I: rBioNet: A COBRA toolbox extension for reconstructing high-quality biochemical networks. Bioinformatics 2011 27:2009-2010

25. UniProt C: Update on activities at the Universal Protein Resource (UniProt) in 2013. Nucleic Acids Res 2013, 41:D43-D47.

26. Chagoyen M, Pazos F: MBRole: enrichment analysis of metabolomic data. Bioinformatics 2011, 27:730-731.

27. Roberts SB, Gowen CM, Brooks JP, Fong SS: Genome-scale metabolic analysis of Clostridium thermocellum for bioethanol production. BMC Syst Biol 2010, 4:31-0509-0504-0531.

28. Roberts SB, Robichaux JL, Chavali AK, Manque PA, Lee V, Lara AM, Papin JA, Buck GA: Proteomic and network analysis characterize stage-specific metabolism in Trypanosoma cruzi. BMC Syst Biol 2009, 3:52-0509-0503-0552.

29. Vanee N, Roberts SB, Fong SS, Manque P, Buck GA: A genome-scale metabolic model of Cryptosporidium hominis. Chem Biodivers 2010, 7:1026-1039.

30. Brooks JP, Burns WP, Fong SS, Gowen CM, Roberts SB: Gap detection for genome-scale constraint-based models. Adv Bioinformatics 2012, 2012:323472.

31. Joyce AR, Palsson BÃ: Toward Whole Cell Modeling And Simulation: Comprehensive Functional Genomics Through The Constraint-Based Approach. Prog Drug Res 2007, 64:267-309.

32. Burgard AP, Pharkya P, Maranas CD: Optknock: a bilevel programming framework for identifying gene knockout strategies for microbial strain optimization. Biotechnol Bioeng 2003, 84:647-657.

33. Ranganathan S, Suthers PF, Maranas CD: OptForce: an optimization procedure for identifying all genetic manipulations leading to targeted overproductions. PLoS Comput Biol 2010, 6:e1000744.

34. Yang L, Cluett WR, Mahadevan R: EMILiO: a fast algorithm for genome-scale strain design. Metab Eng 2011, 13:272-281.

35. Palsson BÃ: Systems Biology: Properties Of Reconstructed Networks. New York Cambridge University Press; 2007.

36. Overbeek R, Begley T, Butler RM, Choudhuri JV, Chuang HY, Cohoon M, de Crecy-Lagard V, Diaz N, Disz T, Edwards R, Fonstein M, Frank ED, Gerdes S, Glass EM, Goesmann A, Hanson A, Iwata-Reuyl D, Jensen R, Jamshidi N, Krause L, Kubal M, Larsen N, Linke B, McHardy AC, Meyer F, Neuweger H, Olsen G, Olson R, Osterman A, Portnoy V, et al: The subsystems approach to genome annotation and its use in the project to annotate 1000 genomes. Nucleic Acids Res 2005, 33:5691-5702.

37. Wilson DB, Kostylev M: Cellulase processivity. Meth Mol Biol 2012, 908:93-99.

38. Henry CS, DeJongh M, Best AA, Frybarger PM, Linsay B, Stevens RL: High-throughput generation, optimization and analysis of genome-scale metabolic models. Nat Biotechnol 2010, 28:977-982.

39. Chandrasekaran S, Price ND: Probabilistic integrative modeling of genome-scale metabolic and regulatory networks in Escherichia coli and Mycobacterium tuberculosis. Proc Natl Acad Sci U S A 2010, 107:17845-17850.

40. Colijn C, Brandes A, Zucker J, Lun DS, Weiner B, Farhat MR, Cheng TY, Moody DB, Murray M, Galagan JE: Interpreting expression data with metabolic flux models: predicting Mycobacterium tuberculosis mycolic acid production. PLoS Comput Biol 2009, 5:e1000489.

41. Lerman JA, Hyduke DR, Latif H, Portnoy VA, Lewis NE, Orth JD, Schrimpe-Rutledge AC, Smith RD, Adkins JN, Zengler K, Palsson BO: In silico method for modelling metabolism and gene product expression at genome scale. Nat Commun 2012, 3:929.

42. Shlomi T, Cabili MN, Herrgard MJ, Palsson BÃ, Ruppin E: Network-based prediction of human tissue-specific metabolism. Nat Biotechnol 2008, 26:1003-1010

43. Gowen CM, Fong SS: Genome-scale metabolic model integrated with RNAseq data to identify metabolic states of Clostridium thermocellum. Biotechnol J 2010, 5:759-767.

44. Orth JD, Conrad TM, Na J, Lerman JA, Nam H, Feist AM, Palsson BO: A comprehensive genome-scale reconstruction of Escherichia coli metabolism-2011. Mol Syst Biol 2011, 7:535

45. Markowitz VM, Chen IM, Palaniappan K, Chu K, Szeto E, Grechkin Y, Ratner A, Jacob B, Huang J, Williams P, Huntemann M, Anderson I, Mavromatis K, Ivanova NN, Kyrpides NC: IMG: the Integrated Microbial Genomes database and comparative analysis system. Nucleic Acids Res 2012, 40:D115-D122.

46. Hyduke DR, Lewis NE, Palsson BO: Analysis of omics data with genome-scale models of metabolism. Mol Biosyst 2013, 9:167-174.

47. Dwivedi RC, Spicer V, Harder M, Antonovici M, Ens W, Standing KG, Wilkins JA, Krokhin OV: Practical implementation of 2D HPLC scheme with accurate peptide retention prediction in both dimensions for high-throughput bottom-up proteomics. Anal Chem 2008, 15;80(18):7036-42.

48. McQueen P, Krokhin O: Optimal selection of $2 \mathrm{D}$ reversed-phase-reversedphase HPLC separation techniques in bottom-up proteomics. Expert Rev Proteomics 2012, 9(2):125-8.

49. Craig $R$, Beavis RC: TANDEM: matching proteins with tandem mass spectra. Bioinformatics 2004, 12;20(9):1466-7.

50. Feist AM, Herrgard MJ, Thiele I, Reed $J$, Palsson BO: Reconstruction of biochemical networks in microorganisms. Nat Rev Microbiol 2009, 7:129-143.

doi:10.1186/s12918-014-0086-2

Cite this article as: Vanee et al:: Proteomics-based metabolic modeling and characterization of the cellulolytic bacterium Thermobifida fusca. BMC Systems Biology 2014 8:86.

\section{Submit your next manuscript to BioMed Central and take full advantage of:}

- Convenient online submission

- Thorough peer review

- No space constraints or color figure charges

- Immediate publication on acceptance

- Inclusion in PubMed, CAS, Scopus and Google Scholar

- Research which is freely available for redistribution

Submit your manuscript at www.biomedcentral.com/submit
C) Biomed Central 